\title{
Frobenius 3-Folds via Singular Flat 3-Webs ${ }^{\star}$
}

Sergey I. AGAFONOV

Departmento de Matemática, Universidade Federal da Paraiba, João Pessoa, Brazil

E-mail: sergey.agafonov@gmail.com

Received May 28, 2012, in final form October 17, 2012; Published online October 21, 2012

http://dx.doi.org/10.3842/SIGMA.2012.078

\begin{abstract}
We give a geometric interpretation of weighted homogeneous solutions to the associativity equation in terms of the web theory and construct a massive Frobenius 3-fold germ via a singular 3 -web germ satisfying the following conditions: 1) the web germ admits at least one infinitesimal symmetry, 2) the Chern connection form is holomorphic, 3) the curvature form vanishes identically.
\end{abstract}

Key words: Frobenius manifold; hexagonal 3-web; Chern connection; infinitesimal symmetry

2010 Mathematics Subject Classification: 53A60; 53D45; 34M35

\section{Introduction}

The theory of Frobenius manifolds, having its origin in theoretical physics, has deep interrelations with apparently very different areas of mathematics: Gromov-Witten invariants and quantum cohomology, integrable systems, singularity, deformation of flat connections etc. We discuss a new aspect of this fruitful and fast developing theory: its relations with the classical chapter of differential geometry, namely the web theory.

The notion of Frobenius manifold, introduced by B. Dubrovin (see [8] and [12]), is a geometric translation of the theory of WDVV-equations that arise originally in the physical context of twodimensional topological field theory.

Definition 1. A Frobenius manifold is a complex analytic manifold $M$ equipped with the following analytic objects:

1) a commutative and associative multiplication on $T_{p} M$,

2) an invariant non-degenerate flat inner product: $\langle u \cdot v, w\rangle=\langle u, v \cdot w\rangle$,

3) a constant unity vector field $e: \nabla e=0, e \cdot v=v \forall v \in T M$,

4) a linear Euler vector field $E: \nabla(\nabla E)=0$,

satisfying the following conditions:

- the flow of $E$ re-scales the multiplication and the inner product,

- 4-tensor $\left(\nabla_{z} c\right)(u, v, w)$ is symmetric in $u, v, w, z$, where

$$
c(u, v, w):=\langle u \cdot v, w\rangle
$$

In this definition, the symbol $\nabla$ stands for the Levi-Civita connection of the inner product $\langle$,$\rangle .$

\footnotetext{
*This paper is a contribution to the Special Issue "Geometrical Methods in Mathematical Physics". The full collection is available at http://www.emis.de/journals/SIGMA/GMMP2012.html
} 
The following geometric construction of 3 -web via Frobenius 3 -fold was proposed in [1]. Consider a massive Frobenius 3 -fold $M$, which means that the algebra $T_{p} M$ is semi-simple for each $p \in U$ for some open set $U \subset M$. Then $T_{p} M$ is the direct product of one-dimensional algebras spanned by idempotents

$$
T_{p} M=\mathbb{C}\left\{e_{1}\right\} \otimes \mathbb{C}\left\{e_{2}\right\} \otimes \mathbb{C}\left\{e_{3}\right\}, \quad e_{i} \cdot e_{j}=\delta_{i j} e_{i} .
$$

In this setting, the unity vector field is

$$
e=e_{1}+e_{2}+e_{3}
$$

Let $S$ be a surface transverse to the unit vector field $e$, then 3 planes spanned by $\left\{e, e_{i}\right\}$ cut 3 directions on $T_{p} S$ (see Fig. 1 on the left). The integral curves of these direction fields build a flat (or hexagonal) 3-web, i.e., at the points with pairwise distinct web directions, this web is locally biholomorphic to 3 families of parallel lines in the plane. Due to the existence of canonical Dubrovin coordinates (see [9]), the distributions $\left\{e, e_{i}\right\}$ are integrable, integral surfaces of each of the distributions being formed by the integral curves of the unity vector field $e$. Thus for each point in $M$ there are 3 integral surfaces intersecting along such a curve. These surfaces cut $S$ along the constructed web. This justifies the following definition (see also Fig. 1 on the right).
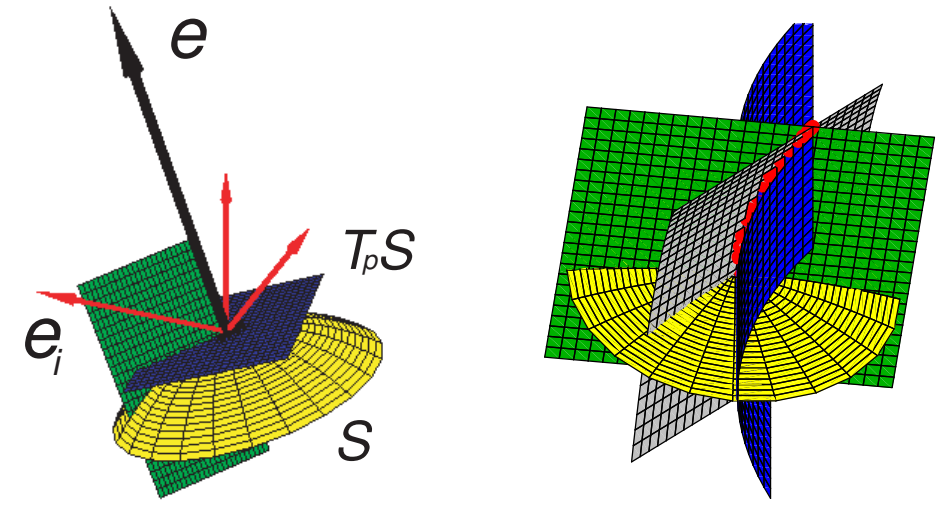

Figure 1. Construction of a booklet 3-webs from Frobenius 3-folds.

Definition 2. The constructed web is called a booklet 3 -web.

The web directions of a booklet 3-web are well-defined everywhere (see [1]), maybe with multiplicity. We call a point regular or non-singular if the web directions at this point are pairwise distinct. The locus of singular points is called the discriminant curve. By definition, a hexagonal 3-web does not have any local invariants at regular points. Its "personality" is encoded in the behavior at singular points, where at least 2 web directions coincide.

A booklet 3-web enjoys the following properties [1]:

- it has at least one infinitesimal symmetry at each singular point,

- its Chern connection form is closed, i.e., the web is flat,

- its Chern connection form is holomorphic at singular points (in a suitable normalization),

- it is biholomorphic to the characteristic 3 -web of the corresponding solution of associativity equation,

- its Chern connection is induced by the connection on $T M$ compatible with the algebraic structure of $M$. 
Note that the existence of a symmetry at a singular point is not a trivial condition for a flat 3-web: even though a flat 3-web has 3-dimensional symmetry algebra at regular points (see [7]), not all symmetries survive at singular ones (see the discussion in [3]). For a booklet 3-web the symmetry is generated by the flow of the Euler field $E$.

The analyticity condition of the Chern connection at singular points is also rather restrictive: a flat 3-web can have closed but not holomorphic connection form. Generically it has a pole on the discriminant curve (see [3] for examples).

For studying our webs we use implicit ODEs; this approach has proved its efficiency in both non-singular (see for instance [11]) and singular cases [3, 4]. Let us consider singular 3 -webs having the first three of the above properties, and suppose that the web directions are well defined (probably, with multiplicities). Then these directions $[d x: d y]$ satisfy a binary differential equation

$$
K_{3}(x, y) d y^{3}+K_{2}(x, y) d y^{2} d x+K_{1}(x, y) d y d x^{2}+K_{0}(x, y) d x^{3}=0,
$$

where the coefficients $K_{i}$ do not vanish simultaneously. Dividing by $d x^{3}$ or $d y^{3}$ one can write it down as an implicit cubic ODE with at least one non-vanishing coefficient. It turns out that such ODEs can be classified. Moreover, the normal form can be effectively computed, whenever the infinitesimal symmetry is known. (See Section 2, where we present the classification results obtained in [3].)

In this paper, we address a natural question: whether it is possible to recover a Frobenius 3 -fold germ starting from a singular 3 -web with the properties mentioned above. Namely we give a construction of a Frobenius 3 -fold germ for each of the normal forms.

The key observation that permitted the classification is that the infinitesimal symmetry operator, vanishing at the base point $(0,0)$ of the web-germ, is a dilatation in suitable coordinates

$$
X=w_{1} x \partial_{x}+w_{2} y \partial_{y}
$$

This gives us two weights $\left[w_{1}: w_{2}\right]$ of the Euler vector field, since, for booklet 3 -webs, the operator $X$ is the projection of the Euler vector field along the unity $e$ to the surface $S$ (see [1]).

Consider the map $\pi_{e}: M \rightarrow S$ that assigns to each $p \in M$ the intersection of its trajectory under the flow of $e$ with $S$. Then the vector fields $e_{i}$ and $v_{i}:=d \pi_{e}\left(e_{i}(p)\right)$ are $\pi$-related: $d \pi_{e}\left(e_{i}(p)\right)=v_{i}\left(\pi_{e}(p)\right)$. Therefore $\left[v_{i}, v_{j}\right]=0$.

If we have a flat 3 -web, then the natural candidates for $v_{i}$ are the commuting web direction vector fields. We recover idempotents in the form $e_{i}=v_{i}+\alpha_{i} \partial_{t}$, where $t$ is a third local coordinate on a Frobenius 3 -fold germ to be constructed. The functions $\alpha_{i}$ are subjected to the following conditions:

1) $\sum e_{i}=\partial_{t}$,

2) $e_{i}$ commute: $\left[e_{i}, e_{j}\right]=0$,

3) there is a non-degenerate flat metric $g$ with $g\left(e_{i}, e_{j}\right)=0, i \neq j$,

4) the flow of $X+w_{3} t \partial_{t}$ re-scales the metric for a suitable weight $w_{3}$.

(By metric we understand a non-degenerate inner product.) It turns out that these conditions can be satisfied, the analyticity of the Chern connection form being crucial for the existence of the metric $g$. We define the multiplication by formula (1). The obtained structure is that of Frobenius 3 -fold since the above conditions on $\alpha_{i}$ are equivalent to the associativity equation with dilatation symmetry.

Thus weighted homogeneous solutions to the associativity equation, i.e. invariant with respect to a dilatation symmetry, can be interpreted geometrically also in terms of the web theory. Namely they describe 4 -webs $\mathfrak{W}_{4}$ of curves in $\left(\mathbb{C}^{3}, 0\right)$ with the following properties: 
- $\mathfrak{W}_{4}$ is flat,

- one of the web foliations defines a non-vanishing vector field $e$,

- $\mathfrak{W}_{4}$ has a 2-dimensional symmetry algebra $\{e, E\}$, where

$$
[e, E] \wedge e=0 \quad \text { and } \quad e \wedge E \not \equiv 0,
$$

- the Chern connection form of the symmetry reduction of $\mathfrak{W}_{4}$ by $e$ remains holomorphic at singular points.

By the symmetry reduction we understand the construction similar to the construction of booklet 3 -web via $\mathfrak{W}_{4}$ formed by the trajectories of $e_{i}$.

The webs $\mathfrak{W}_{4}$ are recovered with some parametric arbitrariness for particular normal forms. This indicates the need for a better understanding of the webs $\mathfrak{W}_{4}$.

\section{Singularities of booklet webs}

In this section we present the classification obtained in [3]. To distinguish between the normal forms, we need invariants, i.e. objects, preserved by local biholomorphisms.

Obviously, root multiplicity and projectivised weights $\left[w_{1}: w_{2}\right]$ are invariant. There is a subtler invariant. Consider the cross-ratio of the three web directions and the direction defined by the infinitesimal symmetry. This function is well-defined on the complement of the discriminant curve and is constant along the trajectories of the symmetry flow. Thus it is a function of a first integral of the symmetry operator. The limit value of this cross-ratio is our third invariant. As the cross-ratio is dependent on the order of its arguments, we use the following symmetrized form: multiply cubic form (2) with a 1-form vanishing on the trajectories of the symmetry group (for normal forms it is $w_{x} x d y-w_{y} y d x$ ), write the resulting quartic form $a_{4} d y^{4}+4 a_{3} d y^{3} d x+6 a_{2} d y^{2} d x^{2}+4 a_{1} d y d x^{3}+a_{0} d x^{4}$, compute $i:=a_{0} a_{4}-4 a_{1} a_{3}+3 a_{2}^{2}$ and $j:=a_{4} a_{2} a_{0}+2 a_{1} a_{2} a_{3}-a_{2}^{3}-a_{4} a_{1}^{2}-a_{0} a_{3}^{2}$. Then the invariant is $\left[i^{3}: j^{2}\right]$. The polynomials $i, j$ are well-known in the classical invariant theory, being invariants of the weights 4 and 6 respectively.

Theorem 1 ([3]). Suppose ODE (2) admits an infinitesimal symmetry $X$ vanishing at the point $(0,0)$ on the discriminant curve and the germ of the Chern connection form is exact $\gamma=d(f)$, where $f$ is some function germ. Then the equation and the symmetry are equivalent to one of the following normal forms:
1) $y^{m_{0}} p^{3}-p=0$,
$X=\left(2+m_{0}\right) x \partial_{x}+2 y \partial_{y}$,
2) $p^{3}+2 x p+y=0$,
$X=2 x \partial_{x}+3 y \partial_{y}$,
3) $\left(p-\frac{2}{3} x\right)\left(p^{2}+\frac{2}{3} x p+y-\frac{2}{9} x^{2}\right)=0$,
$X=x \partial_{x}+2 y \partial_{y}$,
4) $p^{3}+4 x\left(y-\frac{4}{9} x^{3}\right) p+y^{2}+\frac{64}{81} x^{6}-\frac{32}{9} y x^{3}=0$,
$X=x \partial_{x}+3 y \partial_{y}$,
5) $p^{3}+x y^{2} p+\frac{2}{\sqrt{27}} \frac{x^{\frac{3}{2}} y^{3}}{\tan \left(\frac{4}{\sqrt{3}} x^{\frac{3}{2}}\right)}=0$,
$X=y \partial_{y}$,
6) $p^{3}+y^{2} p=\frac{2}{\sqrt{27}} y^{3} \tan (2 \sqrt{3} x+L)$,
$X=y \partial_{y}$,
7) $p^{3}+y^{3+m_{0}} p+y^{\frac{9+3 m_{0}}{2}} U\left(\left[\left(m_{0}+1\right)\right] x y^{\frac{1+m_{0}}{2}}\right)=0$,
$X=\left(1+m_{0}\right) x \partial_{x}-2 y \partial_{y}$,
8) $p^{3}+x y^{3+m_{0}} p-\frac{x^{\frac{3}{2}} y^{\frac{9+3 m_{0}}{2}}}{V\left(\left[\frac{2}{3}\left(m_{0}+1\right)\right] x^{\frac{3}{2}} y^{\frac{1+m_{0}}{2}}\right)}=0$,
$X=\left(1+m_{0}\right) x \partial_{x}-3 y \partial_{y}$. 
The function $U(T)$ is defined, with $L=-\frac{2\left(m_{0}+3\right)}{\left(m_{0}+1\right)}$ and suitable constants $C_{1}, C_{2}$, by the relations

$$
\begin{aligned}
& \frac{T}{3 \sqrt{3} L}=\frac{f^{\prime}\left(-\arctan \left(\frac{3 \sqrt{3}}{2} U\right)\right)}{f\left(-\arctan \left(\frac{3 \sqrt{3}}{2} U\right)\right)}, \quad f(z)=\cos ^{-\mu}(z)\left[C_{1} P_{\nu}^{\mu}(\sin z)+C_{2} Q_{\nu}^{\mu}(\sin z)\right], \\
& f\left(-\arctan \left(\frac{3 \sqrt{3}}{2} U(0)\right)\right) \neq 0, \quad f^{\prime}\left(-\arctan \left(\frac{3 \sqrt{3}}{2} U(0)\right)\right)=0 .
\end{aligned}
$$

The initial value of $U$ vanishes $U(0)=0$ if the number $m_{0}$ is even. If $U(0) \neq 0$ one can choose $0 \leq \arg (U(0))<\pi$.

The function $V(T)$ is defined, with $L=-\frac{5 m_{0}+17}{3\left(m_{0}+1\right)}$, by the relations

$$
\frac{1}{3 \sqrt{3} L} T=\frac{f^{\prime}\left(-\arctan \left(\frac{2}{3 \sqrt{3}} V\right)\right)}{f\left(-\arctan \left(\frac{2}{3 \sqrt{3}} V\right)\right)}, \quad f(z)=\sin ^{\mu}(z) P_{\nu}^{\mu}(\cos z) .
$$

In the above formulas, $P_{\nu}^{\mu}(z), Q_{\nu}^{\mu}(z)$ are Legendre's functions for $\mu=\frac{1}{2}\left(1-\frac{1}{3 L}\right), \nu=\frac{1}{2}\left(\frac{1}{L}-1\right)$, $m_{0}$ is non-negative integer, for the form 6$)$ with $L \neq 0$ one can choose $0 \leq \arg (L)<\pi$.

The weights $\left[w_{1}: w_{2}\right]$, the root multiplicity and the invariant $\left[i^{3}: j^{2}\right]$ uniquely determine the normal form.

Remark 1. The invariant $\left[i^{3}: j^{2}\right]$ assumes the value $[0: 1]$ for the form 5$)$, the value $\left[1: \frac{\tan ^{2}(L)}{-27}\right]$ for the form 6$)$, and the values $\left[1: \frac{U^{2}(0)}{-4}\right],[0: 1]$ for the forms 7$\left.), 8\right)$ respectively.

\section{Metric in flat coordinates}

In this section we present forms of invariant metrics for our Frobenius 3-folds in flat coordinates. For the case when all the weights of the Euler vector fields are distinct, they were obtained for arbitrary $n=3$ in [8]. Here we consider a bit weaker hypothesis that the weights of $x$ and $y$ are not equal. We are looking for a constant matrix

$$
g=\left(\begin{array}{lll}
g_{t t} & g_{t x} & g_{t y} \\
g_{t x} & g_{x x} & g_{x y} \\
g_{t y} & g_{x y} & g_{y y}
\end{array}\right)
$$

where $g_{t t}=\langle e, e\rangle=\left\langle\partial_{t}, \partial_{t}\right\rangle, g_{t x}=\left\langle\partial_{t}, \partial_{x}\right\rangle, \ldots, g_{y y}=\left\langle\partial_{y}, \partial_{y}\right\rangle$, satisfying $L_{E}(g)=$ const $\cdot g$. (The local flow $\exp (E a)$ re-scales the metric.) One has $L_{E}(g)_{t t}=-2 w_{t} g_{t t}, L_{E}(g)_{t x}=\left(-w_{t}-w_{x}\right) g_{t x}$, $\ldots, L_{E}(g)_{y y}=-2 w_{y} g_{y y}$. Therefore the non-vanishing entries in $g$ must be of the same weight. Observe also that for each 3-web in the classification list the inequality $w_{x} \neq w_{y}$ holds true.

- Metric with $\langle e, e\rangle=0$. As $w_{t x} \neq w_{t y}$ and the metric $g$ is non-degenerate exactly one of the the entries $g_{t x}, g_{t y}$ is non-vanishing. Suppose that $g_{t y}=1$. Now $g_{x x} \neq 0$ since $g$ is nondegenerate. This implies $g_{x y}=0, g_{y y}=0$ and $2 w_{x}=w_{t}+w_{y}$. If $g_{t y}=0$ then, similarly, one normalizes $g_{t x}=1$ and obtains $g_{y y} \neq 0, g_{x x}=g_{x y}=0$.

Suppose $g_{x x}=\delta \neq 0$ then $\left\langle e_{1}, e_{2}\right\rangle=\left\langle e_{1}, e_{3}\right\rangle=0$ implies that the weights of $p_{i}$ are zero. This is not possible for our singular webs. Therefore $g_{x x}$ must vanish and the form of the matrix $g$ is

$$
g=\left(\begin{array}{lll}
0 & 1 & 0 \\
1 & 0 & 0 \\
0 & 0 & \delta
\end{array}\right)
$$

where $\delta \neq 0$. 
- Metric with $\langle e, e\rangle \neq 0$. At least one of the entries $g_{t x}, g_{t y}$ vanishes. Let $g_{t y}=0$. Now exactly one of the the entries $g_{x y}, g_{y y}$ is non-vanishing. The entry $g_{x y}$ can not vanish; otherwise $g_{y y} \neq 0, g_{x x}=0, w_{t}=w_{y}$ and because of the non-degeneracy of $g$ we have $g_{t x} \neq 0$, which implies $w_{t}=w_{x}$ and, finally, one obtains a contradiction $w_{x}=w_{y}$. Thus we have $g_{x y} \neq 0, g_{y y}=0$, which implies $g_{x x}=0$. If $g_{t x} \neq 0$ then $w_{t}=w_{x}$. And again we get the contradiction $w_{x}=w_{y}$. Hence one can suppose that the form of the metric is

$$
g=\left(\begin{array}{lll}
\delta & 0 & 0 \\
0 & 0 & 1 \\
0 & 1 & 0
\end{array}\right)
$$

\section{Idempotents}

In this section we determine idempotents $e_{i}$ using the following 2 facts:

1) the metric, being invariant, is diagonal in the basis $\left\{e_{1}, e_{2}, e_{3}\right\}$,

2) the idempotents commute.

Suppose that the following implicit cubic ODE defines our 3-web:

$$
p^{3}+S(x, y) p^{2}+A(x, y) p+B(x, y)=0 .
$$

Let $p_{1}, p_{2}, p_{3}$ be the roots of $(4)$ at a point $(x, y)$ outside the discriminant curve. The following 1 -forms vanish on the solutions

$$
\begin{aligned}
& \sigma_{1}=\left(p_{2}-p_{3}\right)\left(d y-p_{1} d x\right), \\
& \sigma_{2}=\left(p_{3}-p_{1}\right)\left(d y-p_{2} d x\right), \\
& \sigma_{3}=\left(p_{1}-p_{2}\right)\left(d y-p_{3} d x\right)
\end{aligned}
$$

and satisfy

$$
\sigma_{1}+\sigma_{2}+\sigma_{3}=0 \text {. }
$$

Let us introduce an "area" form by

$$
\Omega=\sigma_{1} \wedge \sigma_{2}=\sigma_{2} \wedge \sigma_{3}=\sigma_{3} \wedge \sigma_{1}=\left(p_{1}-p_{2}\right)\left(p_{2}-p_{3}\right)\left(p_{3}-p_{1}\right) d y \wedge d x
$$

The Chern connection form is defined as (see [6])

$$
\gamma:=h_{2} \sigma_{1}-h_{1} \sigma_{2}=h_{3} \sigma_{2}-h_{2} \sigma_{3}=h_{1} \sigma_{3}-h_{3} \sigma_{1}
$$

where $h_{i}$ verify the relations

$$
d \sigma_{i}=h_{i} \Omega .
$$

The web is flat iff the connection form is closed: $d(\gamma)=0$. This implies $d \sigma_{i}=\gamma \wedge \sigma_{i}$ and gives an integrating factor $k$ of the forms $\sigma_{i}$ as a solution to the following equation

$$
d k=-\gamma k \text {. }
$$

Computing the Chern connection form in terms of roots $p_{i}$ and using the Viete formulas one gets

$$
\gamma=\frac{\gamma_{1} d x+\gamma_{2} d y}{-D},
$$


where

$$
\begin{aligned}
\gamma_{1}= & \left(4 B S^{2}-3 A B-S A^{2}\right) S_{x}+B(S A-9 B) S_{y}+\left(2 A^{2}-6 S B\right) A_{x} \\
& +2 B\left(3 A-S^{2}\right) A_{y}+(9 B-S A) B_{x}+\left(A S^{2}-4 A^{2}+3 S B\right) B_{y}, \\
\gamma_{2}= & \left(6 S B-2 A^{2}\right) S_{x}-2 B\left(3 A-S^{2}\right) S_{y}+(S A-9 B) A_{x} \\
& +\left(4 A^{2}-A S^{2}-3 S B\right) A_{y}+\left(6 A-2 S^{2}\right) B_{x}+\left(2 S^{3}+18 B-8 S A\right) B_{y},
\end{aligned}
$$

and $D$ is the discriminant of (4) with respect to $p$

$$
D=18 S A B+S^{2} A^{2}-4 A^{3}-27 B^{2}-4 B S^{3} .
$$

We also need to know how the connection form in the normalization (5) is transformed when we change the local coordinates

$$
\bar{y}=f(x, y), \quad \bar{x}=g(x, y) .
$$

One finds without difficulty that the forms $\overline{\sigma_{i}}$ in the new coordinates are related to the forms in old ones by

$$
\bar{\sigma}_{i}=\frac{\left(f_{y} g_{x}-f_{x} g_{y}\right)^{2} \sigma_{i}}{g_{x}^{3}-S g_{x}^{2} g_{y}+A g_{x} g_{y}^{2}-B g_{y}^{3}} .
$$

Therefore (see [6])

$$
\bar{\gamma}=\gamma+d \ln \left(\frac{\left(f_{y} g_{x}-f_{x} g_{y}\right)^{2}}{g_{x}^{3}-S g_{x}^{2} g_{y}+A g_{x} g_{y}^{2}-B g_{y}^{3}}\right) .
$$

Let $v_{i}=\xi_{i} \partial_{x}+\eta_{i} \partial_{y}$ be commuting multi-valued vector fields, whose trajectories are the web leaves. They may have singularities at singular points: with $K$ satisfying $\frac{d K}{K}=\gamma$ we have

$$
v_{1}=\frac{K\left(\partial_{x}+p_{1} \partial_{y}\right)}{\left(p_{3}-p_{1}\right)\left(p_{1}-p_{2}\right)}, \quad v_{2}=\frac{K\left(\partial_{x}+p_{2} \partial_{y}\right)}{\left(p_{1}-p_{2}\right)\left(p_{2}-p_{3}\right)}, \quad v_{3}=\frac{K\left(\partial_{x}+p_{3} \partial_{y}\right)}{\left(p_{2}-p_{3}\right)\left(p_{3}-p_{1}\right)}
$$

Here $x, y$ are restrictions on $S$ of flat coordinates in $M$, in which the Euler vector field has the form $E=w_{t} t \partial_{t}+w_{x} x \partial_{x}+w_{y} y \partial_{y}$. A subtle point is that flat coordinates are not necessarily the coordinates used for the normal forms: they are related to them by some coordinate transform preserving $X$.

The idempotents are

$$
e_{1}=\alpha \partial_{t}+v_{1}, \quad e_{2}=\beta \partial_{t}+v_{2}, \quad e_{1}=(1-\alpha-\beta) \partial_{t}+v_{3},
$$

where the components $\alpha, \beta$ are to be defined by the orthogonality condition

$$
\left\langle e_{1}, e_{2}\right\rangle=\left\langle e_{2}, e_{3}\right\rangle=\left\langle e_{3}, e_{1}\right\rangle=0 .
$$

- Metric with $\langle e, e\rangle=0$. Equations (8) give

$$
\delta=-1 / K \text {. }
$$

Adjusting the integration constant so that $K=-1$ we have $\delta=1$ and

$$
\alpha=\frac{p_{2} p_{3}-p_{1}\left(p_{2}+p_{3}\right)}{2\left(p_{1}-p_{2}\right)\left(p_{1}-p_{3}\right)}, \quad \beta=\frac{p_{1} p_{3}-p_{2}\left(p_{1}+p_{3}\right)}{2\left(p_{2}-p_{1}\right)\left(p_{2}-p_{3}\right)} .
$$

Since $d K=0$, we see that $\gamma_{1}=\gamma_{2}=0$. The equation $\left[e_{1}, e_{2}\right]=0$ reads as $v_{1}(\beta)=v_{2}(\alpha)$ and obviously implies $\left[e_{1}, e_{3}\right]=\left[e_{2}, e_{3}\right]=0$. The above 3 equations $\gamma_{1}=\gamma_{2}=v_{1}(\beta)-v_{2}(\alpha)=0$ can be resolved with respect to $S_{x}, A_{x}, B_{x}$ to give the following system of hydrodynamic type

$$
S_{x}=\frac{1}{2} A_{y}, \quad A_{x}=2 B y, \quad B_{x}=S B_{y}+B S_{y}-\frac{1}{2} A A_{y} .
$$


Lemma 1. Suppose that the solution web of ODE (4) satisfies the following conditions:

1) it admits an infinitesimal symmetry $X=w_{x} x \partial_{x}+w_{y} y \partial_{y}$ with $w_{x} \neq w_{y}$,

2) the coefficients $S, A, B$ satisfy the system of PDEs (11).

Then there is a Frobenius 3-fold germ with $\langle e, e\rangle=0$ whose booklet web is the solution web of (4).

Proof. Equations (11) ensures the local existence of a function $f(x, y)$ satisfying $f_{y y y}=S$, $f_{y y x}=\frac{1}{2} A, f_{y x x}=B, f_{x x x}=B S-\frac{1}{4} A^{2}$. Thus we obtain the associativity equation

$$
f_{x x x}=f_{y y y} f_{y x x}-f_{y y x}^{2} .
$$

(See [13] where the equivalence of (11) and (12) was established and [2] where equations of these types were studied.) Since the web admits an infinitesimal symmetry $X$, the function $f$ is weighted homogeneous and defines a germ of Frobenius 3-fold with $\langle e, e\rangle=0$ (see [8]). Equation (4) defines the corresponding characteristic web of the above associativity equation. Hence the web is the booklet web of the constructed Frobenius 3-fold germ (see [1]).

The value of the lemma is that it makes unnecessary the checking the potentiality condition for the 4-tensor $\left(\nabla_{z} c\right)(u, v, w)$ in Definition 1 .

Theorem 2. Suppose that the solution web of ODE (4) satisfies the following conditions:

1) it admits an infinitesimal symmetry $X=w_{x} x \partial_{x}+w_{y} y \partial_{y}$ with $w_{x} \neq w_{y}$,

2) its Chern connection form vanishes identically $\gamma=0$,

3) the vector fields $e_{1}, e_{2}$ commute, where $\alpha, \beta$ are as in (10).

Then there is a Frobenius 3-fold germ with $\langle e, e\rangle=0$ whose booklet web is the solution web of (4).

Proof. Define a metric by (3) with $\delta=1$, the multiplication as the direct sum of onedimensional algebras with unities (7), and the Euler vector field by $E=w_{t} t \partial_{t}+X$ with $w_{t}=2 w_{y}-w_{x}$. Then Lemma 1 ensures that the defined structure is that of Frobenius 3-fold.

- Metric with $\langle e, e\rangle \neq 0$. Equations (8) give

$$
\alpha=\frac{\left(p_{1}+p_{2}\right)\left(p_{1}+p_{3}\right)}{\left(p_{1}-p_{2}\right)\left(p_{1}-p_{3}\right)}, \quad \beta=\frac{\left(p_{2}+p_{1}\right)\left(p_{2}+p_{3}\right)}{\left(p_{2}-p_{1}\right)\left(p_{2}-p_{3}\right)},
$$

and

$$
\delta=\frac{-K^{2}}{\left(p_{1}+p_{2}\right)\left(p_{2}+p_{3}\right)\left(p_{3}+p_{1}\right)} .
$$

Vieta formulas result in

$$
\delta=\frac{K^{2}}{S A-B} .
$$

Adjusting the integration constant so that $K=-1$ we have $\delta=1$, provided the condition $2 \frac{d K}{K}=\frac{d(S A-B)}{S A-B}$ is satisfied. Substituting $\frac{d K}{K}=\gamma$ we obtain

$$
2 \gamma=\frac{d(S A-B)}{S A-B}
$$


Together with the commutativity condition $v_{1}(\beta)=v_{2}(\alpha)$, where $\alpha, \beta$ are given now by (13), equation (15) gives the following system of hydrodynamic type

$$
\begin{aligned}
& S_{x}=\frac{\left(A^{2}-A S^{2}+2 S B\right) S_{y}+\left(S^{3}-A S+2 B\right) A_{y}-\left(A+S^{2}\right) B_{y}}{2 A S-2 B}, \\
& A_{x}=\frac{-A S_{y}+S A_{y}+B_{y}}{2}, \\
& B_{x}=\frac{\left(A^{3}+4 B^{2}-3 A S B\right) S_{y}+\left(2 A B-S A^{2}+B S^{2}\right) A_{y}+\left(2 A S^{2}-3 S B-A^{2}\right) B_{y}}{2 A S-2 B} .
\end{aligned}
$$

Lemma 2. Suppose that the solution web of ODE (4) satisfies the following conditions:

1) it admits an infinitesimal symmetry $X=w_{x} x \partial_{x}+w_{y} y \partial_{y}$ with $w_{x} \neq w_{y}$,

2) the coefficients $S, A, B$ of (4) satisfy the system of PDEs (16).

Then there is a Frobenius 3-fold germ with $\langle e, e\rangle=1$ whose booklet web is the solution web of (4).

Proof. It repeats that of Lemma 1. System (16) again is reducible (see [2]) and therefore is equivalent to the local existence of a function $f(x, y)$ satisfying $\frac{f_{y y x}}{f_{y y y}}=S,-\frac{f_{y x x}}{f_{y y y}}=A,-\frac{f_{x x x}}{f_{y y y}}=B$, and the associativity equation

$$
f_{x x x} f_{y y y}-f_{x x y} f_{x y y}=1,
$$

which corresponds to the case $\langle e, e\rangle=1$ (see [8]).

Theorem 3. Suppose that the solution web of ODE (4) satisfies the following conditions:

1) it admits an infinitesimal symmetry $X=w_{x} x \partial_{x}+w_{y} y \partial_{y}$ with $w_{x} \neq w_{y}$,

2) its Chern connection form verifies (15),

$3)$ the vector fields $e_{1}, e_{2}$ commute, where $\alpha, \beta$ are as in (13).

Then there is a Frobenius 3-fold germ with $\langle e, e\rangle=1$ whose booklet web is the solution web of (4).

Proof. The proof is similar to that of Theorem 2. The only difference is that the weight $w_{t}$ now is $w_{t}=\left(w_{x}+w_{y}\right) / 2$.

Remark 2. Equations (9) and (14) imply that the Chern connection form of the booklet web remains holomorphic at singular points: this is straightforward for equation (9), for (14) one has to take into account the associativity equation (17) and, if equation (2) is not monic, the formula (6). Thus, a geometrical interpretation of the analyticity of the connection is the existence of non-degenerate invariant flat metric.

\section{Webs with one elliptic symmetry operator}

We call a symmetry operator $X=w_{x} x \partial_{x}+w_{y} y \partial_{y}$ elliptic if the weight ratio $w_{x} / w_{y}$ is positive. In this section we study the Frobenius 3 -fold germs corresponding to the normal forms 2), 3) and 4) in Theorem 1. The connection form $\gamma$ vanishes identically for each of these equations. The coordinate transformations, preserving the symmetry generator $X$, are non-trivial only for the forms 3) and 4). Up to an unessential scaling, they read as

$$
\bar{x}=x, \quad \bar{y}=y+r x^{2}, \quad r=\text { const },
$$


and

$$
\bar{x}=x, \quad \bar{y}=y+r x^{3}, \quad r=\text { const },
$$

respectively.

Proposition 1. The booklet 3-web of a massive Frobenius 3-fold with $\langle e, e\rangle=1$ cannot have singularities of types 2), 3), 4) in Theorem 1.

Proof. The coefficients $\bar{S}, \bar{A}, \bar{B}$ of the transformed ODEs vanish at singular points. The condition $\gamma \equiv 0$ is also preserved by transformations (18) and (19). This yields a contradiction: $\frac{1}{\delta}=\frac{\bar{S} \bar{A}-\bar{B}}{K^{2}}=0$.

Definition $3([8])$. Two Frobenius manifolds $M$ and $\tilde{M}$ are equivalent if there exists a diffeomorphism

$$
\varphi: M \rightarrow \tilde{M}
$$

being a linear conformal transformation of the corresponding invariant metrics

$$
\varphi^{*} \tilde{g}=c^{2} g
$$

( $c$ is a non-zero constant) with the differential acting as an isomorphism on the tangent algebras

$$
d \varphi: T_{p} M \rightarrow T_{\varphi(p)} \tilde{M} .
$$

Proposition 2. Each of the 3-web germs of type 2), 3), 4) in Theorem 1 is a booklet 3-web for some Frobenius 3-fold germ with $\langle e, e\rangle=0$. Moreover, this germ is unique up to the equivalence of Frobenius 3-folds.

Proof. We use Lemma 1. The coefficients of ODE 2) satisfy conditions (11). Now let us apply transformation (18) to ODE 3) and transformation (19) to ODE 4). Then the first equation of (11) gives $r=-\frac{1}{12}$ for 3 ) and $r=-\frac{1}{9}$ for 4 ), the second and the third equations (11) being satisfied for the found values of $r$. The constructed germ is obviously unique up to an unessential scaling, i.e., up to the equivalence.

Remark 3. The Frobenius 3-folds in the above Proposition are those corresponding to the polynomial solutions of WDVV equation (12) (see [8]), which, of course, is not surprising. The above Frobenius 3 -fold germ can be also recovered directly via Theorem 2 .

\section{Web with a parabolic symmetry operator}

We call a symmetry operator $X=w_{x} x \partial_{x}+w_{y} y \partial_{y}$ parabolic if one of the weights $w_{x}, w_{y}$ vanishes. In this section we study the Frobenius 3 -fold germs corresponding to the normal forms 5) and 6) of Theorem 1. Coordinate transformations, preserving the symmetry generator $X$, are of the form

$$
\bar{y}=y F(x), \quad \bar{x}=G(x),
$$

where

$$
F(0) \neq 0, \quad G^{\prime}(0) \neq 0, \quad G(0)=0 .
$$

Proposition 3. The booklet 3-web of a massive Frobenius 3-fold with $\langle e, e\rangle=1$ cannot have singularities of type 5) or 6 ) of Theorem 1. 
Proof. The orthogonality condition (8) yields the contradiction

$$
\frac{1}{\delta}=-\left.\frac{B F^{3}+2 y A F^{2} F^{\prime}+8 y^{3}\left(F^{\prime}\right)^{3}}{K^{2} F^{4} G^{\prime}}\right|_{(0,0)}=0 .
$$

Proposition 4. There is a one-parameter family of inequivalent Frobenius 3-fold germs with $\langle e, e\rangle=0$, whose booklet web germs are diffeomorphic to 3-web germ 5) or 6) of Theorem 1.

Proof. Consider the following cubic ODE

$$
p^{3}+y s(x) p^{2}+y^{2} a(x) p+y^{3} b(x)=0 .
$$

Equations (11) read as

$$
s^{\prime}=a, \quad a^{\prime}=6 b, \quad b^{\prime}=4 a b-a^{2} .
$$

The invariant $\left[i^{3}: j^{2}\right]$ is equal to $\left[108\left(s^{2}-3 a\right)^{3}:\left(9 a s-2 s^{3}-27 b\right)^{2}\right]$. Adjusting the initial conditions $s(0), a(0), b(0)$ to verify

$$
\left.\frac{\left(9 a s-2 s^{3}-27 b\right)^{2}}{\left(3 a-s^{2}\right)^{3}}\right|_{x=0}=4 \tan ^{2}(L)
$$

and applying Lemma 1 one gets 2-parameter family Frobenius 3-fold germs with the booklet web 6). For the form 5) one chooses $s^{2}-\left.3 a\right|_{x=0}=0$ and $9 a s-2 s^{3}-\left.27 b\right|_{x=0} \neq 0$. Now the symmetry $y \partial_{y}$ reduces the number of parameters in the family of inequivalent Frobenius 3-fold germs to one.

Remark 4. Frobenius 3-fold germ can be also constructed directly via Theorem 2. Consider, for instance, the case 6$)$. The connection form here is $\gamma=\frac{2}{\sqrt{3}} \tan (2 \sqrt{3} x+L) d x$, therefore $K=\frac{1}{\sqrt[3]{\cos (2 \sqrt{3} x+L)}}$. The orthogonality conditions (8) give

$$
\delta=-\frac{G^{\prime}}{K F^{2}},
$$

and the following expressions for the idempotent components

$$
\begin{aligned}
& \alpha=\frac{\left(p_{2} p_{3}-p_{1} p_{2}-p_{1} p_{3}\right) F^{2}-2 y p_{1} F F^{\prime}-y^{2}\left(F^{\prime}\right)^{2}}{2 F^{2}\left(p_{1}-p_{2}\right)\left(p_{1}-p_{3}\right)}, \\
& \beta=\frac{\left(p_{1} p_{3}-p_{2} p_{1}-p_{2} p_{3}\right) F^{2}-2 y p_{2} F F^{\prime}-y^{2}\left(F^{\prime}\right)^{2}}{2 F^{2}\left(p_{2}-p_{1}\right)\left(p_{2}-p_{3}\right)} .
\end{aligned}
$$

Now the commutativity condition $\left[e_{1}, e_{2}\right]=0$ amounts to the following equation for $F$

$$
3 F F^{\prime \prime}-6\left(F^{\prime}\right)^{2}-2 \sqrt{3} \tan (2 \sqrt{3} x+L) F F^{\prime}+F^{2}=0 .
$$

Due to Theorem 2, each solution G, F of equations (20) and (21) defines a Frobenius 3-fold germ.

Remark 5. Substitution $\frac{F^{\prime}}{F}=u$ reduces equation (21) to some Riccati equation. Hence (21) is linearizable. 


\section{Web with a hyperbolic symmetry operator}

We call a symmetry operator $X=w_{x} x \partial_{x}+w_{y} y \partial_{y}$ hyperbolic if the weight ratio $w_{x} / w_{y}$ is negative. In this section we study the Frobenius 3-fold germs corresponding to the normal forms 7) and 8) of Theorem 1. The connection form here is holomorphic, i.e., the factor $K$ does not vanish. Coordinate transformations, preserving the symmetry generator $X$, are of the form

$$
\bar{y}=y Q(s), \quad \bar{x}=x R(s),
$$

where $Q(0) \neq 0, R(0) \neq 0, s=x y^{r}, r=\frac{1+m_{0}}{2}$, and the functions $Q, R$ are even for even $m_{0}$.

Proposition 5. The booklet 3-webs of a massive Frobenius 3-fold with $\langle e, e\rangle=1$ cannot have singularities of types 7 ) or 8 ) of Theorem 1.

Proof. Since the Jacobian matrix of transformation (22) is diagonal at $(0,0)$, we infer that the transformed cubic ODE remains monic with coefficients $\bar{S}, \bar{A}, \bar{B}$ vanishing at $(0,0)$. Further, the factor $\bar{K}$ remains analytic and finite due to (6). Now the condition (14) gives a contradiction

$$
\frac{1}{\delta}=\frac{\bar{S} \bar{A}-\bar{B}}{\bar{K}^{2}}=0
$$

To prove that each singularity of types 7) and 8) can be realized by some booklet web, we

need some properties of the coefficients $\bar{S}, \bar{A}, \bar{B}$. One easily finds by direct calculation the following formulae for them

$$
\bar{S}=\frac{y}{x} \sigma(s), \quad \bar{A}=\frac{y^{2}}{x^{2}} \alpha(s), \quad \bar{B}=\frac{y^{3}}{x^{3}} \beta(s),
$$

where

$$
\begin{aligned}
\sigma(s)= & -\frac{s}{\Delta}\left[3 s^{2}\left(1+r^{2} s^{2}+r^{3} F s^{3}\right) Q^{\prime}\left(R^{\prime}\right)^{2}+r s^{3}(2+3 r F s) Q\left(R^{\prime}\right)^{2}\right. \\
& \left.+2 s\left(3+r^{2} s^{2}\right) R Q^{\prime} R^{\prime}+2 r s^{2} R Q R^{\prime}+3 R^{2} Q^{\prime}\right], \\
\alpha(s)= & \frac{s^{2}}{\Delta}\left[R Q^{2}+\left(3+r^{2} s^{2}\right) R\left(Q^{\prime}\right)^{2}+3 s\left(1+r^{2} s^{2}+r^{3} F s^{3}\right)\left(Q^{\prime}\right)^{2} R^{\prime}\right. \\
& \left.+2 r s^{2}(2+3 r F s) Q Q^{\prime} R^{\prime}+s(1+3 r F s) Q^{2} R^{\prime}+2 r s R Q Q^{\prime}\right], \\
\beta(s)= & -\frac{s^{3}}{\Delta}\left[r s(2+3 r F s) Q\left(Q^{\prime}\right)^{2}+\left(1+r^{2} s^{2}+r^{3} F s^{3}\right)\left(Q^{\prime}\right)^{3}+(1+3 r F s) Q^{2} Q^{\prime}+F Q^{3}\right], \\
\Delta= & R^{3}+3 s R^{2} R^{\prime}+s^{2}\left(3+r^{2} s^{2}\right) R\left(R^{\prime}\right)^{2}+s^{3}\left(1+r^{2} s^{2}+r^{3} F s^{3}\right)\left(R^{\prime}\right)^{3} .
\end{aligned}
$$

Proposition 6. For each 3-web germ singularity of type 7) with odd $m_{0}$ or type 8) of Theorem 1 there is a one-parameter family of inequivalent Frobenius 3 -fold germs with $\langle e, e\rangle=0$, whose booklet web germs are diffeomorphic to this web. For each 3-web germ singularity of type 7) with even $m_{0}$ there is, up to equivalence, unique Frobenius 3 -fold germ with $\langle e, e\rangle=0$.

Proof. Theorem 2 is not of much use here: calculations become very involved. We prove the existence using Lemma 1. Coefficients $S, A, B$ of equation (4) have the form (23). Then equations (24) imply

$$
\sigma(s)=s \tilde{\sigma}(s), \quad \alpha(s)=s^{2} \tilde{\alpha}(s), \quad \beta(s)=s^{3} \tilde{\beta}(s) .
$$

Now the system (11) gives

$$
\tilde{\sigma}^{\prime}=\frac{(k+1) \tilde{\alpha}-k(k+1) s(\tilde{\sigma} \tilde{\alpha}-3 \tilde{\beta})+k^{2}(k+1) s^{2} \tilde{\sigma} \tilde{\beta}}{1-k s \tilde{\sigma}+k^{2} s^{2} \tilde{\alpha}-k^{3} s^{3} \tilde{\beta}},
$$




$$
\begin{aligned}
& \tilde{\alpha}^{\prime}=\frac{6(k+1) \tilde{\beta}-2 k(k+1) s\left(\tilde{\alpha}^{2}-\tilde{\sigma} \tilde{\beta}\right)+2 k^{2}(k+1) s^{2} \tilde{\alpha} \tilde{\beta}}{1-k s \tilde{\sigma}+k^{2} s^{2} \tilde{\alpha}-k^{3} s^{3} \tilde{\beta}}, \\
& \tilde{\beta}^{\prime}=\frac{-(k+1)\left(\tilde{\alpha}^{2}-4 \tilde{\sigma} \tilde{\beta}\right)-2 k(k+1) s \tilde{\alpha} \tilde{\beta}+3 k^{2}(k+1) s^{2} \tilde{\beta}^{2}}{1-k s \tilde{\sigma}+k^{2} s^{2} \tilde{\alpha}-k^{3} s^{3} \tilde{\beta}} .
\end{aligned}
$$

This system has solutions for each choice of initial conditions $\tilde{\sigma}(0), \tilde{\alpha}(0), \tilde{\beta}(0)$. In terms of $\tilde{\sigma}(0)$, $\tilde{\alpha}(0), \tilde{\beta}(0)$ the invariant $\left[i^{3}: j^{2}\right]$ reads as

$$
\left[108\left\{\tilde{\sigma}^{2}(0)-3 \tilde{\alpha}(0)\right\}^{3}:\left\{2 \tilde{\sigma}^{3}(0)-9 \tilde{\alpha}(0) \tilde{\sigma}(0)+27 \tilde{\beta}(0)\right\}^{2}\right] .
$$

For the form 8$)$ we set $\tilde{\alpha}(0)=\tilde{\sigma}^{2}(0) / 3$, and choose $\tilde{\sigma}(0), \tilde{\beta}(0)$ to satisfy $\tilde{\sigma}^{3}(0) \neq 27 \tilde{\beta}^{2}(0)$. The symmetry generated by the Euler vector field reduces the number of free parameters by one, therefore we get one-parameter family of inequivalent 3 -fold germs.

For the form 7) consider first the case of odd $m_{0}$. Adjusting the initial conditions so that the value of the invariant coincides with the corresponding value of the normal form, and taking into account the symmetry, we obtain again one-parameter family of inequivalent 3-fold germs.

Now suppose that $m_{0}$ is even. System (25) is symmetric with respect to the following involution: $s \rightarrow-s, \tilde{\sigma} \rightarrow-\tilde{\sigma}, \tilde{\alpha} \rightarrow \tilde{\alpha}, \tilde{\beta} \rightarrow-\tilde{\beta}$. Uniqueness of the solution of Cauchy problem with initial conditions $\tilde{\sigma}(0)=0, \tilde{\alpha}(0)=a_{0}, \tilde{\beta}(0)=0$ ensures that the function $\tilde{\sigma}$ and $\tilde{\beta}$ are odd, while $\tilde{\alpha}$ is even. Therefore the coefficients

$$
S=\frac{y}{x} s \tilde{\sigma}(s), \quad A=\frac{y^{2}}{x^{2}} s^{2} \tilde{\alpha}(s), \quad B=\frac{y^{3}}{x^{3}} s^{3} \tilde{\beta}(s)
$$

of ODE (4) are holomorphic and satisfy (11). Here the structure of Frobenius 3 -fold is unique up to the equivalence generated by the Euler vector field.

\section{Web with 2-dimensional symmetry algebra}

In this section we consider the form 1). Here the symmetry algebra is 2-dimensional: note that the equation 1 ) is invariant with respect to the translation operator $\partial_{x}$. For a Frobenius 3 -fold to be constructed we have an alternative: either the Euler vector field vanishes at $(0,0)$ or it does not vanish.

Proposition 7. The booklet 3-web germ of a massive Frobenius 3-fold cannot have singularities of type 1) of Theorem 1, if the projection of the Euler vector field to the surface $S$ along the unity vector e vanishes.

Proof. The symmetry operator giving rise to the Euler vector field is

$$
\left(1+m_{0} / 2\right) x \partial_{x}+y \partial_{y}
$$

Then the flat coordinates are, up to an unessential scaling

$$
\bar{x}=x+c y^{1+m_{0} / 2}, \quad \bar{y}=y .
$$

The orthogonality condition (8) yields: $\delta=0$ for $\langle e, e\rangle=0$ and $\frac{1}{\delta}=0$ for $\langle e, e\rangle \neq 0$.

Unfortunately, because of computational difficulties, the author is not able to provide a complete analysis for the case when the projection of $E$ to $S$ does not vanish.

Proposition 8. For each 3-web germ singularity of type 1) there is parametric family of inequivalent Frobenius 3-fold germs with $\langle e, e\rangle=0$, whose booklet web germs are diffeomorphic to this web. 
Proof. The proof is somewhat of an "experimental" nature: the author used the symbolic computational system Maple.

Let us choose the symmetry operator as $X=(1+(l+1) x) \partial_{x}+y \partial_{y}$ and $1+(l+1) x$ as a new coordinate $z$. Now the base point of the 3 -fold germ is $(1,0)$. We are looking for the flat coordinates in the form

$$
\bar{x}=z^{\alpha} R(t), \quad \bar{y}=z^{\beta} Q(t), \quad t=y z^{-\frac{1}{1+m_{0} / 2}}
$$

with analytic $R$ and $Q$ subjected to $\alpha R Q^{\prime}-\left.\beta Q R^{\prime}\right|_{0} \neq 0$.

The orthogonality condition (8) implies that necessarily $\alpha=2 \beta-1$ for $\langle e, e\rangle=0$. The analysis of ODEs for $R$ and $S$, arising from the hypotheses of Theorem 2, shows that they have at least local solutions at $t=0$ for any $\beta \neq \frac{1}{2}$. Therefore one can construct at least 1 -parametric family of inequivalent Frobenius 3-fold germs.

Remark 6. The symmetry operator in the flat coordinates is $(2 \beta-1) z \partial_{z}+\beta y \partial_{y}$. Observe that $\beta$ is not necessarily rational, unlike the case of triple root.

\section{Concluding remarks}

1. The 4-web of curves $\mathfrak{W}_{4}$ discussed in Introduction is formed by the trajectories of the unity vector field $e$ and of the vector fields of idempotents $e_{i}$. The generalization to Frobenius manifolds of higher dimensions is straightforward. We hope that the study of this object, namely, $(n+1)$-web of curves $\mathfrak{W}_{n+1}$ in $n$-dimensional Frobenius manifold $M^{n}$ will provide a better insight into the singular set (or "discriminant") of the Frobenius manifold. Note that for the 3-webs with parabolic and hyperbolic symmetries the 4 -web $\mathfrak{W}_{4}$ are recovered with some parametric arbitrariness. We hope to interpret these parameters in terms of $\mathfrak{W}_{4}$.

It seems that the web of curves, with the exception of webs in the plane, was not a very popular object to study in differential geometry (see surveys [10] and [5]).

2. The reduction of $\mathfrak{W}_{n+1}$ by the symmetry generated by the unity vector field $e$ can also be generalized to higher dimensions. As a result we obtain, for $n$-dimensional Frobenius manifold, a flat $n$-web germ of curves in $\left(\mathbb{C}^{n-1}, 0\right)$ admitting a "linear" symmetry.

3. It is interesting that Frobenius 3 -folds with $\langle e, e\rangle \neq 0$ does not produce singular booklet 3 -webs with triple root and vanishing symmetry. The author does not know if one can get a singular booklet 3 -webs with non-vanishing symmetry at this situation. An approach based

on Lemma 2 gives singular ODEs for the coefficients $S=\frac{y}{x} \tilde{\sigma}(s), A=\frac{y^{2}}{x^{2}} \tilde{\alpha}(s)$, and $B=\frac{y^{3}}{x^{3}} \tilde{\beta}(s)$, if one imposes initial conditions corresponding to the desired singularity.

\section{Acknowledgements}

This research was partially supported by MCT/CNPq/MEC/CAPES - Grant 552758/2011-6.

\section{References}

[1] Agafonov S.I., Flat 3-webs via semi-simple Frobenius 3-manifolds, J. Geom. Phys. 62 (2012), 361-367, arXiv:1108.1997.

[2] Agafonov S.I., Linearly degenerate reducible systems of hydrodynamic type, J. Math. Anal. Appl. 222 (1998), 15-37.

[3] Agafonov S.I., Local classification of singular hexagonal 3-webs with holomorphic Chern connection form and infinitesimal symmetries, arXiv:1105.1402.

[4] Agafonov S.I., On implicit ODEs with hexagonal web of solutions, J. Geom. Anal. 19 (2009), 481-508, arXiv:0808.0348. 
[5] Akivis M.A., Goldberg V.V., Differential geometry of webs, in Handbook of Differential Geometry, Vol. I, North-Holland, Amsterdam, 2000, 1-152.

[6] Blaschke W., Einführung in die Geometrie der Waben, Birkhäuser Verlag, Basel und Stuttgart, 1955.

[7] Cartan E., Les sous-groupes des groupes continus de transformations, Ann. Sci. École Norm. Sup. (3) 25 (1908), 57-194.

[8] Dubrovin B., Geometry of 2D topological field theories, in Integrable Systems and Quantum Groups (Montecatini Terme, 1993), Lecture Notes in Math., Vol. 1620, Springer, Berlin, 1996, 120-348, hep-th/9407018.

[9] Dubrovin B., Integrable systems in topological field theory, Nuclear Phys. B 379 (1992), 627-689.

[10] Grifone J., Salem E. (Editors), Web theory and related topics, World Scientific Publishing Co. Inc., River Edge, NJ, 2001.

[11] Hénaut A., On planar web geometry through abelian relations and connections, Ann. of Math. (2) 159 (2004), 425-445.

[12] Manin Yu.I., Frobenius manifolds, quantum cohomology, and moduli spaces, American Mathematical Society Colloquium Publications, Vol. 47, American Mathematical Society, Providence, RI, 1999.

[13] Mokhov O.I., Ferapontov E.V., Associativity equations of two-dimensional topological field theory as integrable Hamiltonian nondiagonalizable systems of hydrodynamic type, Funct. Anal. Appl. 30 (1996), 195-203, hep-th/9505180. 